Sammlung Metzler

Band 36 


\title{
Joachim Bumke
}

\section{Wolfram von Eschenbach}

\author{
6., neu bearbeitete Auflage
}

Verlag J.B. Metzler

Stuttgart • Weimar 
CIP-Titelaufnahme der Deutschen Bibliothek

Bumke, Joachim:

Wolfram von Eschenbach / Joachim Bumke.

$-6 .$, neu bearb. Aufl.

- Stuttgart : Metzler, 1991

(Sammlung Metzler ; Bd. 36)

ISBN 978-3-476-16036-2

NE: GT

\author{
ISSN 0058-3667 \\ ISBN 978-3-476-16036-2 \\ ISBN 978-3-476-04148-7 (eBook) \\ DOI 10.1007/978-3-476-04148-7
}

SM 36

Dieses Werk einschließlich aller seiner Teile ist urheberrechtlich geschützt. Jede Verwertung außerhalb der engen Grenzen des Urheberrechtsgesetzes ist ohne Zustimmung des Verlages unzulässig und strafbar. Das gilt insbesondere für Vervielfältigungen, Übersetzungen, Mikroverfilmungen und die Einspeicherung und Verarbeitung in elektronischen Systemen.

(C) 1991 Springer-Verlag GmbH Deutschland

Ursprünglich erschienen bei J.B. Metzlersche Verlagsbuchhandlung und Carl Ernst Poeschel Verlag GmbH in Stuttgart 1991 


\section{Vorwort zur 6. Auflage}

Die "Sammlung Metzler « will »Auskünfte über die gesicherten Fakten « geben.Dieses Programm fordert eine kritische Überprüfung des gesamten Wissensstandes, und das ist gerade heute wieder aktuell. Über einen mittelalterlichen Dichter wie Wolfram von Eschenbach ist aber so wenig bekannt, daß eine Darstellung, die sich wirklich auf die gesicherten Fakten beschränken wollte, ganz uninteressant wäre. Man wird daher auch in diesem Bändchen manches Ungesicherte und Hypothetische finden. Ich habe mich bemüht, ein möglichst zuverlässiges Bild vom Stand der Forschung zu geben, habe jedoch angesichts der Vielfalt von Ansätzen und Standpunkten in der neueren Wolframforschung manches übergehen müssen, was vielleicht eine eingehendere Diskussion verdient. $\mathrm{Da}$ es heute kein einheitliches Wolframbild gibt, wie in der theologisch geprägten Forschung nach dem Z weiten Weltkrieg, ist sicherlich nicht negativ zu bewerten.

Die 6. Auflage unterscheidet sich von der fünften durch die Ergänzungen der Literaturangaben und die Revision verschiedener Abschnitte, insbesondere über Wolframs Bildung, seine poetologischen Aussagen und seine Gönner. Neu sind in der 6. Auflage die "Handlungsanalysen « der drei epischen Werke, durch die der Umfang des Bändchens sich fast verdoppelt hat. Dahinter steht die Auffassung, daß der Zugang zu einer erzählenden Dichtung am besten zu finden ist, wenn man sich dem Handlungsgang anvertraut. Die interpretatorischen Akzente, die dabei gesetzt werden, sind als Einladung zu einem Gespräch über die Texte gedacht und sollen zum kritischen Weiterdenken anregen. Das Programm der "Sammlung Metzler « kann auch in diesem Sinn verstanden werden.

Köln, November 1989

J.B. 


\section{Inhalt}

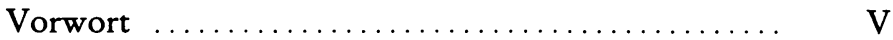

Abkürzungen $\ldots \ldots \ldots \ldots \ldots \ldots \ldots \ldots \ldots \ldots \ldots \ldots \ldots$ IX

I. Der Dichter in seiner Zeit $\ldots \ldots \ldots \ldots \ldots \ldots . . .1$

1. Der Dichter ........................... 1

Herkunft 1 Stand 4 Bildung 7

2. Gönner und Publikum ................... 10

Landgraf Hermann und der Thüringer Hof 10 Der fränkisch-bayerische Gönnerkreis 13

3. Literarische Beziehungen $\ldots \ldots \ldots \ldots \ldots \ldots \ldots . \quad 17$

4. Das Werk .......................... 19

Überblick 19 Chronologie 19 Sprache 21 Metrik 23

Stil 24 Kunstanspruch 25

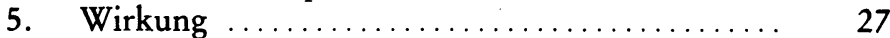

Literatur .............................. 30

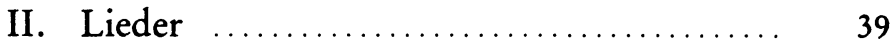

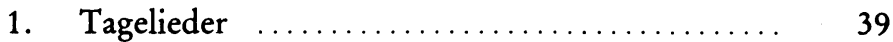

2. Minnelieder $\ldots \ldots \ldots \ldots \ldots \ldots \ldots \ldots \ldots \ldots, \quad 42$

3. Überlieferung und Wirkungsgeschichte ........ 43

Literatur ................................ 44

III. Parzival ............................ 47

1. Handlungsanalyse .................... 47

Der Prolog 47 Die Geschichten von Gahmuret (Buch I-II) 50 Die erste Parzivalpartie (Buch III-VI) 59 Die erste Gawanpartie (Buch VII-VIII) 84 Parzival bei Trevrizent (Buch IX) 91 Die zweite Gawanpartie (Buch X-XIV) 99 Der Abschluß der Parzivalhandlung (Buch XV-XVI) 119

2. Interpretationsprobleme

Religiöse Motive 128 Liebesthematik und Frauenbild 132 Gesellschaftsdarstellung 135 Verwandtschaftsbeziehungen 139 Der Gral 141 
3. Bauform und Erzählhaltung $\ldots \ldots \ldots \ldots \ldots \ldots$

Aufbau 146 Bucheinteilung und Dreißigergliederung 148 Erzählform 150

4. Der Stoff und seine Bearbeitung

Die Parzival-Gral-Sage 153 Wolframs Hauptquelle:

Der >Conte du Graal c von Chrétien de Troyes 156 Die

Nebenquellen 159 Das Kyotproblem 164

5. Entstehung, Überlieferung, Wirkungsgeschichte

Entstehung 168 Überlieferung 170 Wirkung 172

Literatur

IV. Willehalm

1. Handlungsanalyse

Der Prolog 207 Die erste Schlacht auf Alischanz (Buch

I-II) 209 Zwischen den Schlachten (Buch III-VI) 217

Die zweite Schlacht auf Alischanz (Buch VII--X) 232

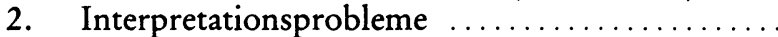

Die Darstellung der höfischen Gesellschaft 242 Politische Motive: Könige, Fürsten, Ministerialität und Stadt 244 Kreuzzugsidee und religiöser Friedensgedanke 247 Das Heidenbild 249

3. Aufbau

Bucheinteilung und Dreißigergliederung 250 Bauform 251 Die Schlußgestaltung 252

4. Der Stoff und seine Bearbeitung

Historische Grundlagen 256 Der französische Epenzyklus um Guillaume d'Orange 257 Wolframs Vorlage: , La Bataille d'Aliscans 258 Die Bearbeitung des Stoffes 259

5. Überlieferung und Wirkungsgeschichte

Handschriftliche Überlieferung 261 Wirkung 262

V. Titurel .............................. 275

1. Handlungsanalyse ...................... 275

Das erste Fragment 275 Das zweite Fragment 279

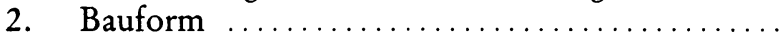

Aufbauplan 283 Metrik und Aufführungsform 284

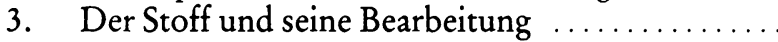

Das Quellenproblem 285 Das Verhältnis zum >Parzival 286

4. Überlieferung und Wirkungsgeschichte ........

Literatur

Register 


\section{Abkürzungen}

$\begin{array}{ll}\text { ABäG } & \text { Amsterdamer Beiträge zur älteren Germanistik } \\ \text { AfdA } & \text { Anzeiger für deutsches Altertum } \\ \text { AG } & \text { Acta Germanica } \\ \text { Akad. d. Wiss. } & \text { Akademie der Wissenschaften } \\ \text { AKG } & \text { Archiv für Kulturgeschichte } \\ \text { altdt. } & \text { altdeutsch } \\ \text { Anz. } & \text { Anzeiger } \\ \text { Bd(e). } & \text { Band, Bände } \\ \text { Beitr. } & \text { Beiträge zur Geschichte der deutschen Sprache } \\ & \text { und Literatur } \\ \text { CCM } & \text { Cahiers de civilisation médiévale } \\ \text { CG } & \text { Colloquia Germanica } \\ \text { Diss. } & \text { Dissertation } \\ \text { dt. } & \text { deutsch } \\ \text { DU. } & \text { Der Deutschunterricht } \\ \text { DVjs. } & \text { Deutsche Vierteljahrsschrift für Literaturwissen- } \\ & \text { schaft und Geistesgeschichte } \\ \text { ebda } & \text { ebenda } \\ \text { ed. } & \text { herausgegeben von } \\ \text { Et. germ. } & \text { Etudes germaniques } \\ \text { Euph. } & \text { Euphorion } \\ \text { GLL } & \text { German Life and Letters } \\ \text { GQ } & \text { German Quarterly } \\ \text { GR } & \text { Germanic Review } \\ \text { GRM } & \text { Germanisch-Romanische Monatsschrift } \\ \text { Habil.schr. } & \text { Habilitationsschrift } \\ \text { hrsg. } & \text { herausgegeben } \\ \text { Hs(s). } & \text { Handschrift(en) } \\ \text { JEGP } & \text { Journal of English and Germanic Philology } \\ \text { Jh. } & \text { Jahrhundert } \\ \text { Lit. Jb. } & \text { Literaturwissenschaftliches Jahrbuch der } \\ & \text { Görres-Gesellschaft } \\ \text { MA } & \text { Medium Aevum } \\ \text { masch. } & \text { maschinenschriftlich } \\ \text { MF } & \text { Minnesangs Frühling } \\ \text { mhd. } & \text { mittelhochdeutsch } \\ \text { MLN } & \text { Modern Language Notes } \\ \text { MLQ } & \text { Modern Language Quarterly } \\ \text { MLR } & \text { Modern Language Review } \\ & \end{array}$


Neoph. Neophilologus

Neuph. Mitt. Neuphilologische Mitteilungen

Parz., Pz. Parzival

Ph. D.

Doctor of Philosophy

phil.-hist. Kl. philologisch-historische Klasse

PhQ

Philological Quarterly

PMLA

Progr.

publ.

RF

Publications of the Modern Language Association of America

s.

SB

St. med.

Programm

herausgegeben

Romanische Forschungen

siehe

Sitzungsbericht(e)

Stud. neoph.

Tit.

Studi medievali

VI

Studia neophilologica

Titurel

VL

Die deutsche Literatur des Mittelalters.

Wh.

WmhM

WvE

WW

ZfdA

ZfdPh.

Verfasserlexikon $\left({ }^{2} \mathrm{VL}=2\right.$. Aufl. $)$

Willehalm

Würzburger medizinhistorische Mitteilungen

Wolfram von Eschenbach

Wirkendes Wort

Zeitschrift für deutsches Altertum und deutsche

Literatur

ZfdSp.

$\mathrm{ZfrPh}$.

Zeitschrift für deutsche Philologie

Zeitschrift für deutsche Sprache

Zeitschrift für romanische Philologie 